\title{
Nanophase Separation of Poly( $N$-alkyl acrylamides): The Dependence of the Formation of Lamellar Structures on Their Alkyl Side Chains
}

Kazuki Ebata, ${ }^{\dagger}$ Yuki Hashimoto, ${ }^{\dagger}$ Shunsuke Yamamoto, ${ }^{\ddagger}$ Masaya Mitsuishi,,${ }^{\ddagger}$ Shusaku Nagano, ${ }^{\S}$ and Jun Matsui ${ }^{\perp *}$

${ }^{\dagger}$ Graduate School of Science and Engineering, Yamagata University, 1-4-12 Kojirakawa-machi, Yamagata 990-8560, Japan

施stitute of Multidisciplinary Research for Advanced Materials, Tohoku University, 2-1-1

Katahira, Aoba-ku, Sendai 980-8577, Japan

${ }^{\S}$ Nagoya University Venture Business Laboratory, Nagoya University, Furo-cho, Chikusa, Nagoya 464-8603, Japan

${ }^{\perp}$ Faculty of Science, Yamagata University, 1-4-12 Kojirakawa-machi, Yamagata 990-8560, Japan

*Author to whom correspondence should be addressed; e-mail:jun_m@sci.kj.yamagata-u.ac.jp 
Synthesis of $N$-alkyl acrylamides

$N$-alkyl amine (TCI, Japan) was dissolved in chloroform to the concentration of $0.1 \mathrm{M}$. To this solution, triethylamine (1.2 equivalent to $N$-alkyl amine, TCI, Japan) was added. The solution was cooled at $0{ }^{\circ} \mathrm{C}$ and acryloyl chloride (1.2 equivalent to $N$-alkyl amine) was added dropwise. After addition of acryloyl chloride, the solution was heated to room temperature and reaction was carried out for $3 \mathrm{~h}$. Then the reaction solution was washed with $5 \mathrm{wt} \% \mathrm{HCl}$, saturated $\mathrm{Na}_{2} \mathrm{CO}_{3}$, and saturated $\mathrm{NaCl}$. The organic solution was collected and dried with $\mathrm{Na}_{2} \mathrm{SO}_{4}$. The crude products were purified by vacuum distillation for $n=4\left(0.3\right.$ Torr, $55^{\circ} \mathrm{C}$, ) and $n=5\left(0.3\right.$ Torr, $\left.80^{\circ} \mathrm{C}\right)$ or recrystallization from chloroform-hexane mixed solvent for $n \geq 6$. The purified compounds were characterized with ${ }^{1} \mathrm{H}$ NMR spectra.

butylacrylamide $(n=4)$

$\left.1 \mathrm{H} \mathrm{NMR} \mathrm{(500} \mathrm{MHz,} \mathrm{CDCl}_{3}\right) \delta\left(\delta\right.$ in ppm):6.27 (dd, 1H, $\mathrm{H}_{2} \mathrm{C}=\mathrm{CH}-$ ), 6.11 (dd, 1H, $\left.\mathrm{H} H \mathrm{C}=\mathrm{CH}-\right), 5.85$ (s, $\left.1 \mathrm{H},-\mathrm{NH}-\mathrm{CH}_{2}-\mathrm{CH}_{2}-\right), 5.62(\mathrm{dd}, 1 \mathrm{H}, H \mathrm{HC}=\mathrm{CH}-), 3.33$ (q, 2H, $\left.-\mathrm{NH}-\mathrm{CH}_{2}-\mathrm{CH}_{2}-\right), 1.50-1.56(\mathrm{~m}, 2 \mathrm{H}$, $\left.-\mathrm{NH}-\mathrm{CH}_{2}-\mathrm{CH}_{2}-\mathrm{CH}_{2}-\mathrm{CH}_{3}\right), 1.37$ (m, 2H, $\left.-\mathrm{NH}-\mathrm{CH}_{2}-\mathrm{CH}_{2}-\mathrm{CH}_{2}-\mathrm{CH}_{3}\right), 0.93$ (t, 3H, -NH-CH $2-\mathrm{CH}_{2}-\mathrm{CH}_{2}-$ $\left.\mathrm{CH}_{3}\right)$

pentylacrylamide $(n=5)$

$1 \mathrm{H} \mathrm{NMR}\left(500 \mathrm{MHz}, \mathrm{CDCl}_{3}\right) \delta: 6.27\left(\mathrm{dd}, 1 \mathrm{H}, \mathrm{H}_{2} \mathrm{C}=\mathrm{CH}-\right), 6.11(\mathrm{dd}, 1 \mathrm{H}, \mathrm{HHC}=\mathrm{CH}-), 5.86(\mathrm{~s}, 1 \mathrm{H},-$ $\left.\mathrm{NH}-\mathrm{CH}_{2}-\mathrm{CH}_{2}-\right), 5.62$ (dd, $\left.1 \mathrm{H}, H \mathrm{HC}=\mathrm{CH}-\right), 3.32$ (q, 2H, -NH-CH $\left.\mathrm{H}_{2}-\mathrm{CH}_{2}-\right), 1.51-1.57$ (m, 2H, -NH$\left.\mathrm{CH}_{2}-\mathrm{CH}_{2}-\left(\mathrm{CH}_{2}\right)_{2}-\mathrm{CH}_{3}\right), 1.30-1.36\left(\mathrm{~m}, 4 \mathrm{H},-\mathrm{NH}-\mathrm{CH}_{2}-\mathrm{CH}_{2}-\left(\mathrm{CH}_{2}\right)_{2}-\mathrm{CH}_{3}\right), 0.90\left(\mathrm{t}, 3 \mathrm{H},-\mathrm{NH}-\mathrm{CH}_{2}-\mathrm{CH}_{2}-\right.$ $\left.\left(\mathrm{CH}_{2}\right)_{2}-\mathrm{CH}_{3}\right)$

hexylacrylamide $(n=6)$

1H NMR (500 MHz, $\left.\mathrm{CDCl}_{3}\right) \delta: 6.26\left(\mathrm{dd}, 1 \mathrm{H}, \mathrm{H}_{2} \mathrm{C}=\mathrm{CH}-\right.$ ), $6.08(\mathrm{dd}, 1 \mathrm{H}, \mathrm{H} H \mathrm{C}=\mathrm{CH}-), 5.63(\mathrm{dd}, 1 \mathrm{H}$, $H \mathrm{HC}=\mathrm{CH}-), 5.55\left(\mathrm{~s}, 1 \mathrm{H},-\mathrm{NH}-\mathrm{CH}_{2}-\mathrm{CH}_{2}-\right), 3.27-3.35\left(\mathrm{~m}, 2 \mathrm{H},-\mathrm{NH}-\mathrm{CH}_{2}-\mathrm{CH}_{2}-\right)$, 1.51-1.56 (m, 2H, $\left.\mathrm{NH}-\mathrm{CH}_{2}-\mathrm{CH}_{2}-\left(\mathrm{CH}_{2}\right)_{3}-\mathrm{CH}_{3}\right), 1.25-1.37\left(\mathrm{~m}, 6 \mathrm{H},-\mathrm{NH}-\mathrm{CH}_{2}-\mathrm{CH}_{2}-\left(\mathrm{CH}_{2}\right)_{3}-\mathrm{CH}_{3}\right), 0.89$ (t, 3H, -NH-CH$\left.\mathrm{CH}_{2}-\left(\mathrm{CH}_{2}\right)_{3}-\mathrm{CH}_{3}\right)$

heptylacrylamide $(n=7)$

1H NMR (500 MHz, $\left.\mathrm{CDCl}_{3}\right) \delta: 6.27\left(\mathrm{dd}, 1 \mathrm{H}, \mathrm{H}_{2} \mathrm{C}=\mathrm{CH}-\right), 6.07$ (dd, 1H, $\left.\mathrm{HHC}=\mathrm{CH}-\right), 5.63(\mathrm{dd}, 1 \mathrm{H}$, $H \mathrm{HC}=\mathrm{CH}-), 5.51\left(\mathrm{~s}, 1 \mathrm{H},-\mathrm{NH}-\mathrm{CH}_{2}-\mathrm{CH}_{2-}\right), 3.33\left(\mathrm{q}, 2 \mathrm{H},-\mathrm{NH}-\mathrm{CH}_{2}-\mathrm{CH}_{2}-\right), 1.53\left(\mathrm{~m}, 2 \mathrm{H},-\mathrm{NH}-\mathrm{CH}_{2}-\right.$ $\left.\mathrm{CH}_{2}-\left(\mathrm{CH}_{2}\right)_{4}-\mathrm{CH}_{3}\right), 1.28-1.33\left(\mathrm{~m}, 8 \mathrm{H},-\mathrm{NH}-\mathrm{CH}_{2}-\mathrm{CH}_{2}-\left(\mathrm{CH}_{2}\right)_{4}-\mathrm{CH}_{3}\right), 0.88$ (t, 3H, $-\mathrm{NH}-\mathrm{CH}_{2}-\mathrm{CH}_{2}-$ $\left.\left(\mathrm{CH}_{2}\right)_{4}-\mathrm{CH}_{3}\right)$ 
octylacrylamide $(n=8)$

$1 \mathrm{H}$ NMR $\left(500 \mathrm{MHz}, \mathrm{CDCl}_{3}\right) \delta: 6.25\left(\mathrm{dd}, 1 \mathrm{H}, \mathrm{H}_{2} \mathrm{C}=\mathrm{CH}-\right), 6.05(\mathrm{dd}, 1 \mathrm{H}, \mathrm{H} H \mathrm{C}=\mathrm{CH}-), 5.61(\mathrm{dd}, 1 \mathrm{H}$, $H \mathrm{HC}=\mathrm{CH}-), 5.47\left(\mathrm{~s}, 1 \mathrm{H},-\mathrm{NH}-\mathrm{CH}_{2}-\mathrm{CH}_{2}-\right), 3.26-3.33$ (m, 2H, -NH-CH$\left.{ }_{2}-\mathrm{CH}_{2}-\right)$, 1.49-1.59 (m, 2H, $\left.\mathrm{NH}-\mathrm{CH}_{2}-\mathrm{CH}_{2}-\left(\mathrm{CH}_{2}\right)_{5}-\mathrm{CH}_{3}\right), 1.18-1.31$ (m, 10H, $\left.-\mathrm{NH}-\mathrm{CH}_{2}-\mathrm{CH}_{2}-\left(\mathrm{CH}_{2}\right)_{5}-\mathrm{CH}_{3}\right), 0.86$ (t, 3H, -NH-CH $2-$ $\left.\mathrm{CH}_{2}-\left(\mathrm{CH}_{2}\right)_{5}-\mathrm{CH}_{3}\right)$

nonylacrylamide $(n=9)$

$1 \mathrm{H}$ NMR (500 MHz, $\left.\mathrm{CDCl}_{3}\right) \delta: 6.27\left(\mathrm{dd}, 1 \mathrm{H}, \mathrm{H}_{2} \mathrm{C}=\mathrm{CH}-\right), 6.07(\mathrm{dd}, 1 \mathrm{H}, \mathrm{HHC}=\mathrm{CH}-), 5.63(\mathrm{dd}, 1 \mathrm{H}$, $H \mathrm{HC}=\mathrm{CH}-), 5.49\left(\mathrm{~s}, 1 \mathrm{H},-\mathrm{NH}-\mathrm{CH}_{2}-\mathrm{CH}_{2}-\right), 3.33\left(\mathrm{q}, 2 \mathrm{H},-\mathrm{NH}-\mathrm{CH}_{2}-\mathrm{CH}_{2}-\right), 1.56\left(\mathrm{~m}, 2 \mathrm{H},-\mathrm{NH}-\mathrm{CH}_{2}-\right.$ $\left.\mathrm{CH}_{2}-\left(\mathrm{CH}_{2}\right)_{6}-\mathrm{CH}_{3}\right), 1.26-1.31\left(\mathrm{~m}, 12 \mathrm{H},-\mathrm{NH}-\mathrm{CH}_{2}-\mathrm{CH}_{2}-\left(\mathrm{CH}_{2}\right)_{6}-\mathrm{CH}_{3}\right), 0.88\left(\mathrm{t}, 3 \mathrm{H},-\mathrm{NH}-\mathrm{CH}_{2}-\mathrm{CH}_{2}-\right.$ $\left.\left(\mathrm{CH}_{2}\right)_{6}-\mathrm{CH}_{3}\right)$

decylacrylamide $(n=10)$

$1 \mathrm{H}$ NMR $\left(500 \mathrm{MHz}, \mathrm{CDCl}_{3}\right) \delta: 6.27\left(\mathrm{dd}, 1 \mathrm{H}, \mathrm{H}_{2} \mathrm{C}=\mathrm{CH}-\right), 6.06(\mathrm{dd}, 1 \mathrm{H}, \mathrm{HHC}=\mathrm{CH}-), 5.62(\mathrm{dd}, 1 \mathrm{H}$, $H \mathrm{HC}=\mathrm{CH}-), 5.49\left(\mathrm{~s}, 1 \mathrm{H},-\mathrm{NH}-\mathrm{CH}_{2}-\mathrm{CH}_{2}-\right), 3.32\left(\mathrm{q}, 2 \mathrm{H},-\mathrm{NH}-\mathrm{CH}_{2}-\mathrm{CH}_{2}-\right), 1.51$ (m, 2H, -NH-CH${ }_{2}-$ $\left.\mathrm{CH}_{2}-\left(\mathrm{CH}_{2}\right)_{7}-\mathrm{CH}_{3}\right), 1.25-1.30\left(\mathrm{~m}, 14 \mathrm{H},-\mathrm{NH}-\mathrm{CH}_{2}-\mathrm{CH}_{2}-\left(\mathrm{CH}_{2}\right)_{7}-\mathrm{CH}_{3}\right), 0.87$ (t, 3H, -NH-CH$-\mathrm{CH}_{2}-$ $\left.\left(\mathrm{CH}_{2}\right)_{7}-\mathrm{CH}_{3}\right)$

undecylacrylamide $(n=11)$

$1 \mathrm{H}$ NMR (500 MHz, $\left.\mathrm{CDCl}_{3}\right) \delta: 6.27\left(\mathrm{dd}, 1 \mathrm{H}, \mathrm{H}_{2} \mathrm{C}=\mathrm{CH}-\right), 6.07(\mathrm{dd}, 1 \mathrm{H}, \mathrm{H} H \mathrm{C}=\mathrm{CH}-), 5.63(\mathrm{dd}, 1 \mathrm{H}$, $H \mathrm{HC}=\mathrm{CH}-), 5.49\left(\mathrm{~s}, 1 \mathrm{H},-\mathrm{NH}-\mathrm{CH}_{2}-\mathrm{CH}_{2}-\right), 3.33\left(\mathrm{q}, 2 \mathrm{H},-\mathrm{NH}-\mathrm{CH}_{2}-\mathrm{CH}_{2}-\right), 1.55$ (m, 2H, -NH-CH${ }_{2}-$ $\left.\mathrm{CH}_{2}-\left(\mathrm{CH}_{2}\right)_{8}-\mathrm{CH}_{3}\right), 1.21-1.31\left(\mathrm{~m}, 16 \mathrm{H},-\mathrm{NH}-\mathrm{CH}_{2}-\mathrm{CH}_{2}-\left(\mathrm{CH}_{2}\right)_{8}-\mathrm{CH}_{3}\right), 0.88$ (t, 3H, $-\mathrm{NH}-\mathrm{CH}_{2}-\mathrm{CH}_{2}-$ $\left.\left(\mathrm{CH}_{2}\right)_{8}-\mathrm{CH}_{3}\right)$

dodecylacrylamide $(n=12)$

$1 \mathrm{H} \mathrm{NMR}\left(500 \mathrm{MHz}, \mathrm{CDCl}_{3}\right) \delta: 6.27\left(\mathrm{dd}, 1 \mathrm{H}, \mathrm{H}_{2} \mathrm{C}=\mathrm{CH}-\right), 6.08(\mathrm{dd}, 1 \mathrm{H}, \mathrm{H} H \mathrm{C}=\mathrm{CH}-), 5.63(\mathrm{dd}, 1 \mathrm{H}$, $H \mathrm{HC}=\mathrm{CH}-), 5.53\left(\mathrm{~s}, 1 \mathrm{H},-\mathrm{NH}-\mathrm{CH}_{2}-\mathrm{CH}_{2-}\right), 3.31-3.35\left(\mathrm{~m}, 2 \mathrm{H},-\mathrm{NH}-\mathrm{CH}_{2}-\mathrm{CH}_{2}-\right), 1.50-1.56(\mathrm{~m}, 2 \mathrm{H},-$ $\left.\mathrm{NH}-\mathrm{CH}_{2}-\mathrm{CH}_{2}-\left(\mathrm{CH}_{2}\right)_{9}-\mathrm{CH}_{3}\right), 1.26-1.31\left(\mathrm{~m}, 18 \mathrm{H},-\mathrm{NH}-\mathrm{CH}_{2}-\mathrm{CH}_{2}-\left(\mathrm{CH}_{2}\right)_{9}-\mathrm{CH}_{3}\right), 0.88\left(\mathrm{t}, 3 \mathrm{H},-\mathrm{NH}-\mathrm{CH}_{2}-\right.$ $\left.\mathrm{CH}_{2}-\left(\mathrm{CH}_{2}\right)_{9}-\mathrm{CH}_{3}\right)$ 
tridecylacrylamide $(n=13)$

1H NMR (500 MHz, $\left.\mathrm{CDCl}_{3}\right) \delta: 6.27\left(\mathrm{dd}, 1 \mathrm{H}, \mathrm{H}_{2} \mathrm{C}=\mathrm{CH}-\right), 6.07(\mathrm{dd}, 1 \mathrm{H}, \mathrm{H} H \mathrm{C}=\mathrm{CH}-), 5.63(\mathrm{dd}, 1 \mathrm{H}$, $H \mathrm{HC}=\mathrm{CH}-), 5.50\left(\mathrm{~s}, 1 \mathrm{H},-\mathrm{NH}-\mathrm{CH}_{2}-\mathrm{CH}_{2}-\right), 3.33\left(\mathrm{q}, 2 \mathrm{H},-\mathrm{NH}-\mathrm{CH}_{2}-\mathrm{CH}_{2}-\right), 1.50-1.57$ (m, 2H, -NH$\left.\mathrm{CH}_{2}-\mathrm{CH}_{2}-\left(\mathrm{CH}_{2}\right)_{10}-\mathrm{CH}_{3}\right), 1.21-1.31\left(\mathrm{~m}, 20 \mathrm{H},-\mathrm{NH}-\mathrm{CH}_{2}-\mathrm{CH}_{2}-\left(\mathrm{CH}_{2}\right)_{10}-\mathrm{CH}_{3}\right), 0.88$ (t, $3 \mathrm{H},-\mathrm{NH}-\mathrm{CH}_{2}-$ $\left.\mathrm{CH}_{2}-\left(\mathrm{CH}_{2}\right)_{10}-\mathrm{CH}_{3}\right)$

tetradecylacrylamide $(n=14)$

1H NMR (500 MHz, $\left.\mathrm{CDCl}_{3}\right) \delta: 6.27\left(\mathrm{dd}, 1 \mathrm{H}, \mathrm{H}_{2} \mathrm{C}=\mathrm{CH}-\right), 6.07(\mathrm{dd}, 1 \mathrm{H}, \mathrm{H} H \mathrm{C}=\mathrm{CH}-), 5.63(\mathrm{dd}, 1 \mathrm{H}$, $H \mathrm{HC}=\mathrm{CH}-), 5.49\left(\mathrm{~s}, 1 \mathrm{H},-\mathrm{NH}-\mathrm{CH}_{2}-\mathrm{CH}_{2}-\right), 3.33\left(\mathrm{q}, 2 \mathrm{H},-\mathrm{NH}-\mathrm{CH}_{2}-\mathrm{CH}_{2}-\right), 1.50-1.56(\mathrm{~m}, 2 \mathrm{H},-\mathrm{NH}-$ $\left.\mathrm{CH}_{2}-\mathrm{CH}_{2}-\left(\mathrm{CH}_{2}\right)_{11}-\mathrm{CH}_{3}\right), 1.25-1.31\left(\mathrm{~m}, 22 \mathrm{H},-\mathrm{NH}-\mathrm{CH}_{2}-\mathrm{CH}_{2}-\left(\mathrm{CH}_{2}\right)_{11}-\mathrm{CH}_{3}\right), 0.88\left(\mathrm{t}, 3 \mathrm{H},-\mathrm{NH}-\mathrm{CH}_{2}-\right.$ $\left.\mathrm{CH}_{2}-\left(\mathrm{CH}_{2}\right)_{11}-\mathrm{CH}_{3}\right)$

pentadecylacrylamide $(n=15)$

1H NMR (500 MHz, $\left.\mathrm{CDCl}_{3}\right) \delta: 6.27\left(\mathrm{dd}, 1 \mathrm{H}, \mathrm{H}_{2} \mathrm{C}=\mathrm{CH}-\right), 6.07(\mathrm{dd}, 1 \mathrm{H}, \mathrm{H} H \mathrm{C}=\mathrm{CH}-), 5.63(\mathrm{dd}, 1 \mathrm{H}$, $H \mathrm{HC}=\mathrm{CH}-), 5.49\left(\mathrm{~s}, 1 \mathrm{H},-\mathrm{NH}-\mathrm{CH}_{2}-\mathrm{CH}_{2}-\right), 3.33\left(\mathrm{q}, 2 \mathrm{H},-\mathrm{NH}-\mathrm{CH}_{2}-\mathrm{CH}_{2}-\right), 1.50-1.56(\mathrm{~m}, 2 \mathrm{H},-\mathrm{NH}-$ $\left.\mathrm{CH}_{2}-\mathrm{CH}_{2}-\left(\mathrm{CH}_{2}\right)_{12}-\mathrm{CH}_{3}\right), 1.25-1.31\left(\mathrm{~m}, 24 \mathrm{H},-\mathrm{NH}-\mathrm{CH}_{2}-\mathrm{CH}_{2}-\left(\mathrm{CH}_{2}\right)_{12}-\mathrm{CH}_{3}\right), 0.88\left(\mathrm{t}, 3 \mathrm{H},-\mathrm{NH}-\mathrm{CH}_{2}-\right.$ $\left.\mathrm{CH}_{2}-\left(\mathrm{CH}_{2}\right)_{12}-\mathrm{CH}_{3}\right)$

hexadecylacrylamide $(n=16)$

1H NMR (500 MHz, $\left.\mathrm{CDCl}_{3}\right) \delta: 6.27\left(\mathrm{dd}, 1 \mathrm{H}, \mathrm{H}_{2} \mathrm{C}=\mathrm{CH}-\right), 6.07$ (dd, $\left.1 \mathrm{H}, \mathrm{H} H \mathrm{C}=\mathrm{CH}-\right), 5.63(\mathrm{dd}, 1 \mathrm{H}$, $H \mathrm{HC}=\mathrm{CH}-), 5.49\left(\mathrm{~s}, 1 \mathrm{H},-\mathrm{NH}-\mathrm{CH}_{2}-\mathrm{CH}_{2}-\right), 3.33\left(\mathrm{q}, 2 \mathrm{H},-\mathrm{NH}-\mathrm{CH}_{2}-\mathrm{CH}_{2}-\right), 1.52-1.56$ (m, 2H, -NH$\left.\mathrm{CH}_{2}-\mathrm{CH}_{2}-\left(\mathrm{CH}_{2}\right)_{13}-\mathrm{CH}_{3}\right), 1.25-1.31\left(\mathrm{~m}, 26 \mathrm{H},-\mathrm{NH}-\mathrm{CH}_{2}-\mathrm{CH}_{2}-\left(\mathrm{CH}_{2}\right)_{13}-\mathrm{CH}_{3}\right), 0.88$ (t, 3H, -NH-CH$\left.\mathrm{CH}_{2}-\left(\mathrm{CH}_{2}\right)_{13}-\mathrm{CH}_{3}\right)$

hepthadecylacrylamide $(n=17)$

1H NMR (500 MHz, $\left.\mathrm{CDCl}_{3}\right) \delta: 6.27\left(\mathrm{dd}, 1 \mathrm{H}, \mathrm{H}_{2} \mathrm{C}=\mathrm{CH}-\right), 6.07$ (dd, 1H, $\left.\mathrm{HHC}=\mathrm{CH}-\right), 5.63$ (dd, 1H, $H \mathrm{HC}=\mathrm{CH}-), 5.51\left(\mathrm{~s}, 1 \mathrm{H},-\mathrm{NH}-\mathrm{CH}_{2}-\mathrm{CH}_{2}-\right), 3.33\left(\mathrm{q}, 2 \mathrm{H},-\mathrm{NH}-\mathrm{CH}_{2}-\mathrm{CH}_{2}-\right), 1.53\left(\mathrm{~m}, 2 \mathrm{H},-\mathrm{NH}-\mathrm{CH}_{2}-\right.$ $\left.\mathrm{CH}_{2}-\left(\mathrm{CH}_{2}\right)_{14}-\mathrm{CH}_{3}\right), 1.25-1.31\left(\mathrm{~m}, 28 \mathrm{H},-\mathrm{NH}-\mathrm{CH}_{2}-\mathrm{CH}_{2}-\left(\mathrm{CH}_{2}\right)_{14}-\mathrm{CH}_{3}\right), 0.88$ (t, 3H, -NH-CH$-\mathrm{CH}_{2}-$ $\left.\left(\mathrm{CH}_{2}\right)_{14}-\mathrm{CH}_{3}\right)$

octadecylacrylamide $(n=18)$

1H NMR (500 MHz, $\left.\mathrm{CDCl}_{3}\right) \delta: 6.27\left(\mathrm{dd}, 1 \mathrm{H}, \mathrm{H}_{2} \mathrm{C}=\mathrm{CH}-\right), 6.07$ (dd, 1H, $\left.\mathrm{HHC}=\mathrm{CH}-\right), 5.63$ (dd, 1H, $H \mathrm{HC}=\mathrm{CH}-), 5.49\left(\mathrm{~s}, 1 \mathrm{H},-\mathrm{NH}-\mathrm{CH}_{2}-\mathrm{CH}_{2}-\right) 3.33$ (q, 2H, $\left.-\mathrm{NH}-\mathrm{CH}_{2}-\mathrm{CH}_{2}-\right), 1.50-1.58\left(\mathrm{~m}, 2 \mathrm{H},-\mathrm{NH}-\mathrm{CH}_{2}-\right.$ 
$\left.\mathrm{CH}_{2}-\left(\mathrm{CH}_{2}\right)_{15}-\mathrm{CH}_{3}\right), 1.22-1.31\left(\mathrm{~m}, 30 \mathrm{H},-\mathrm{NH}-\mathrm{CH}_{2}-\mathrm{CH}_{2}-\left(\mathrm{CH}_{2}\right)_{15}-\mathrm{CH}_{3}\right), 0.88$ (t, 3H, -NH-CH $2-\mathrm{CH}_{2}-$ $\left.\left(\mathrm{CH}_{2}\right)_{15}-\mathrm{CH}_{3}\right)$

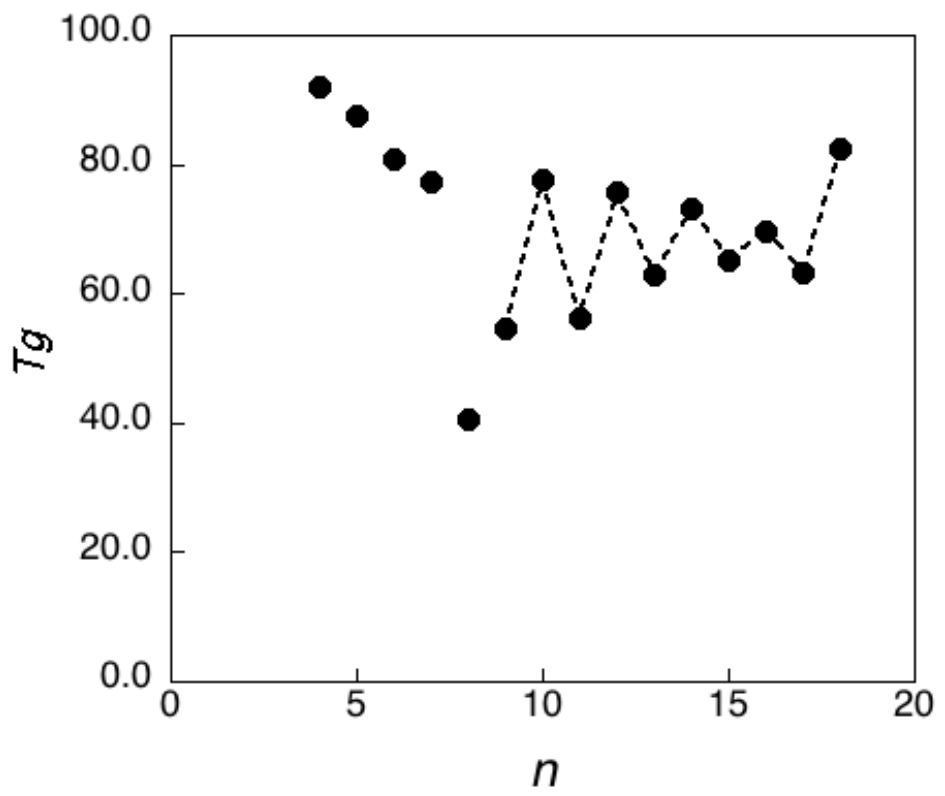

Figure S1. Tg for $\mathrm{p}(\mathrm{AlkylAms)}$. The dotted line is a guide for the eye.

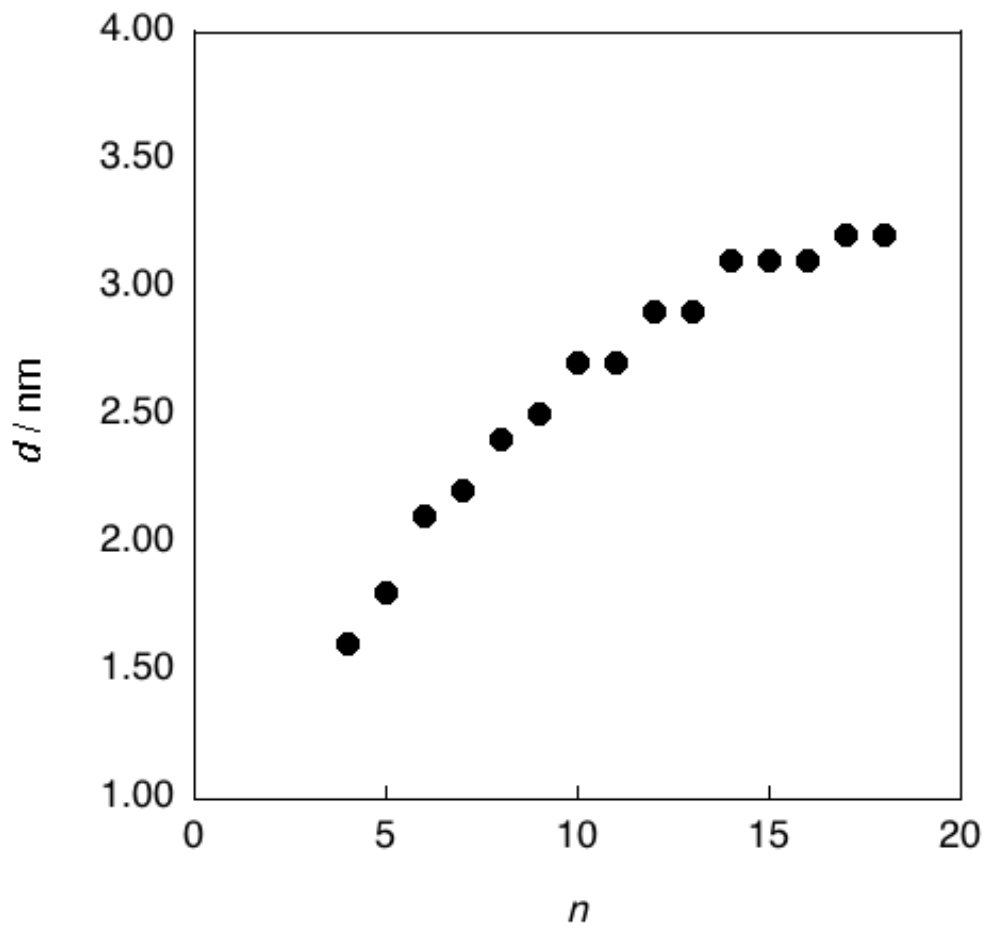

Figure S2. Dependence of alkyl nanodomain sizes with $n$. 


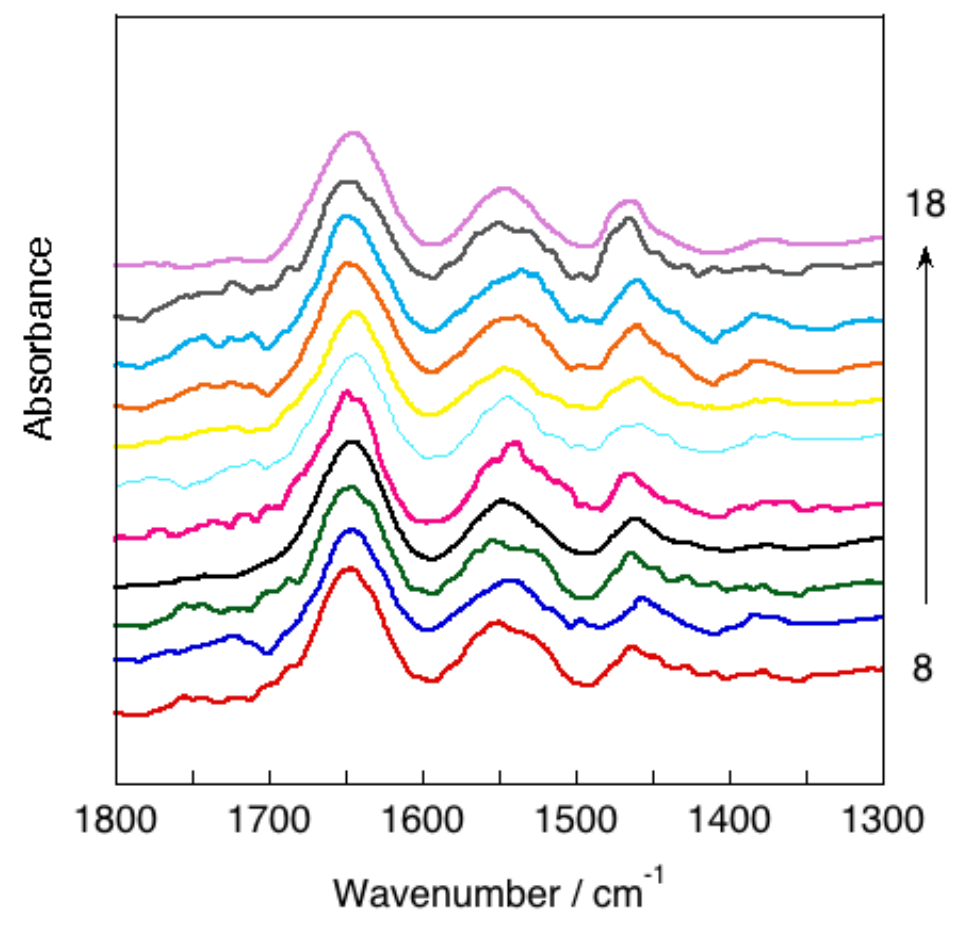

Figure S3. FT-IR spectra of lamellar film of p(AlkylAms) $(n=8-18)$ at the amide region. 

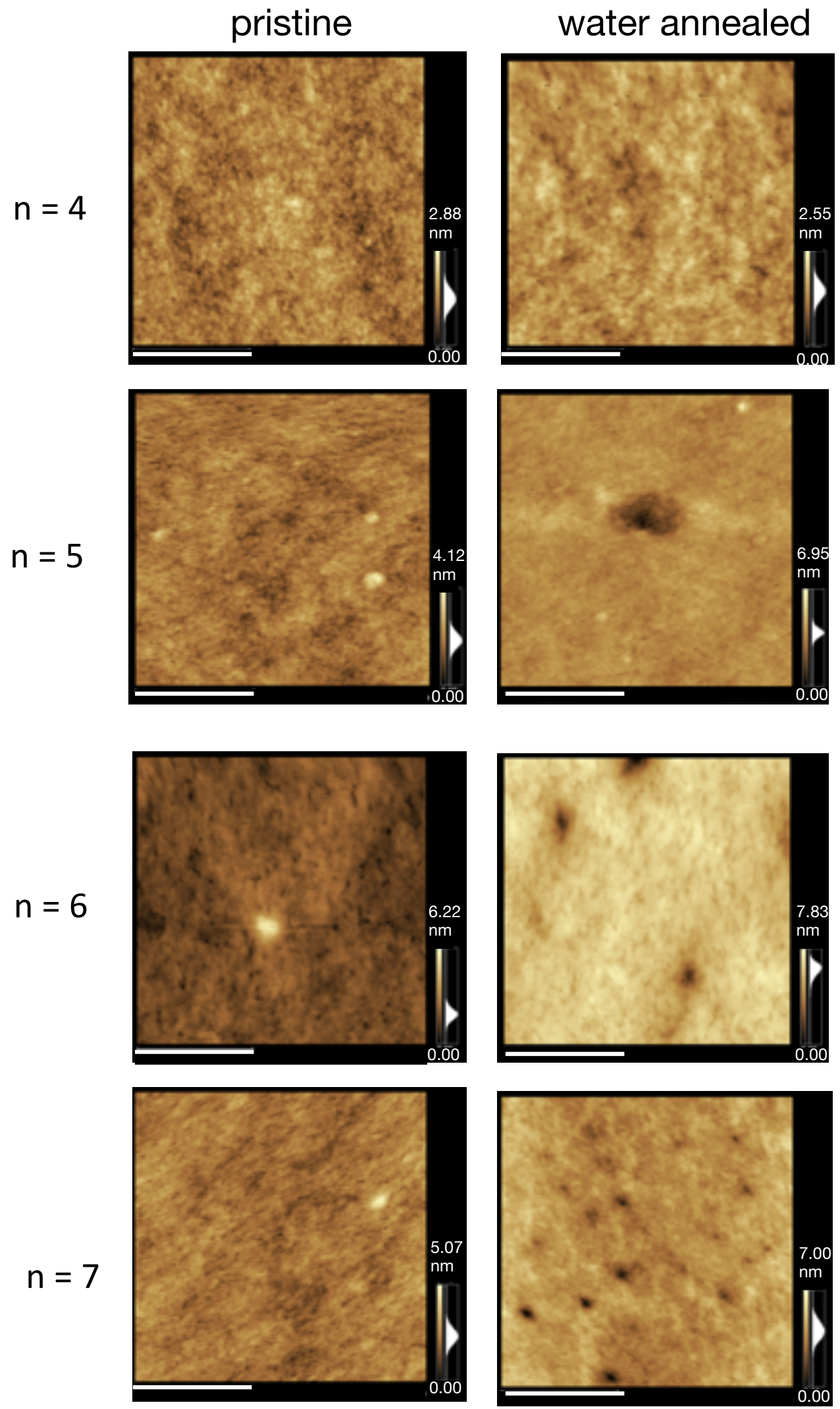

Figure S4 AFM images for $\mathrm{p}($ AlkylMA). Left pristine and right water annealed film. White scale bar in the images indicate $200 \mathrm{~nm}$ 


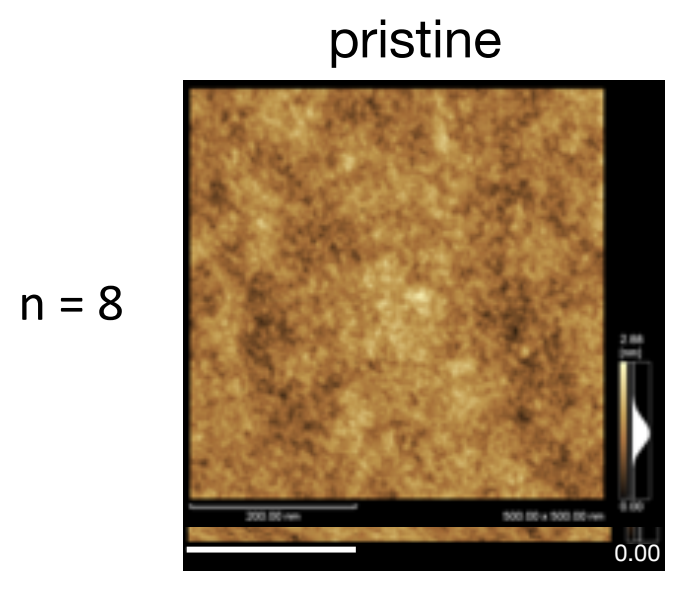

water annealed
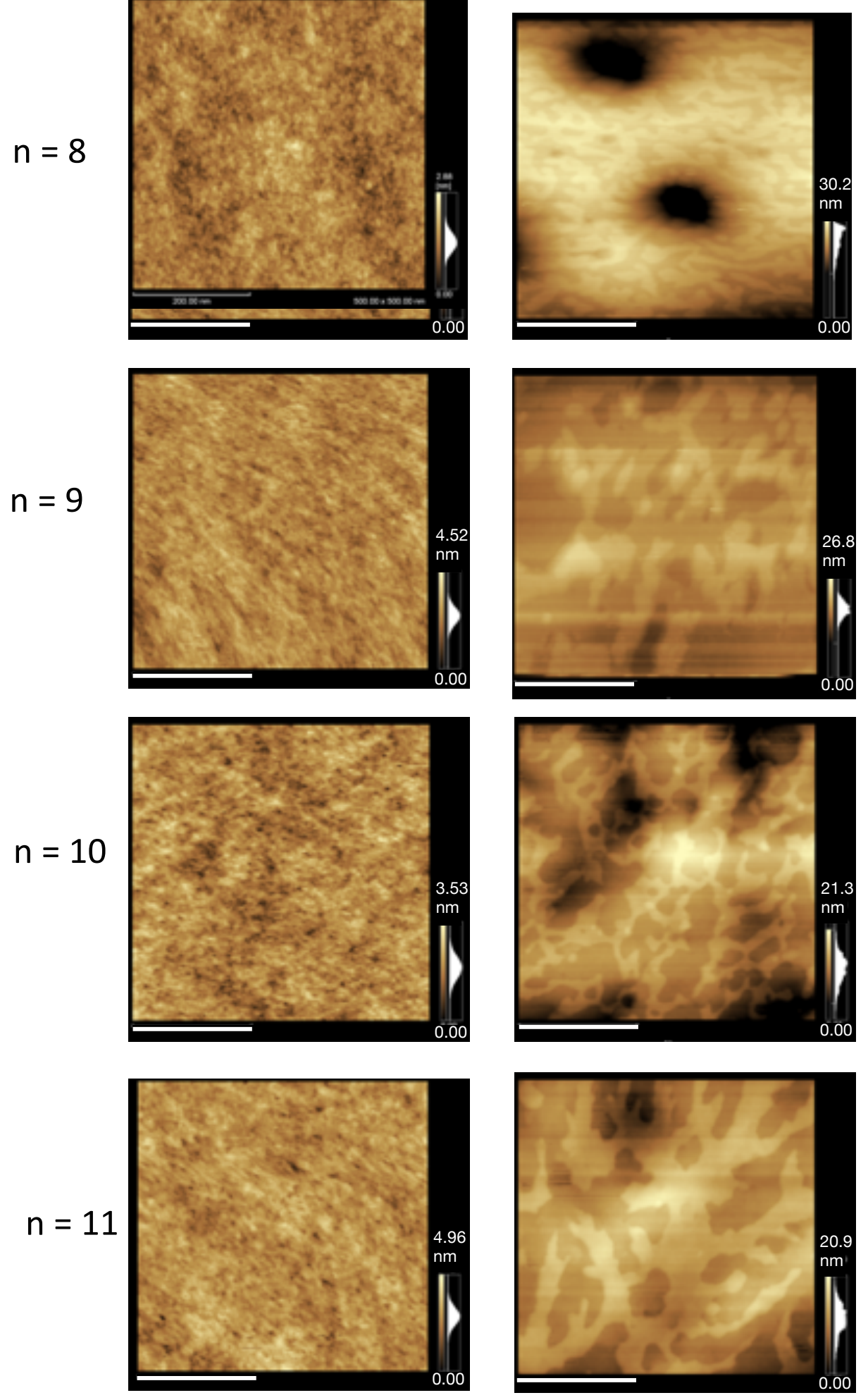

Figure S4 Continued 


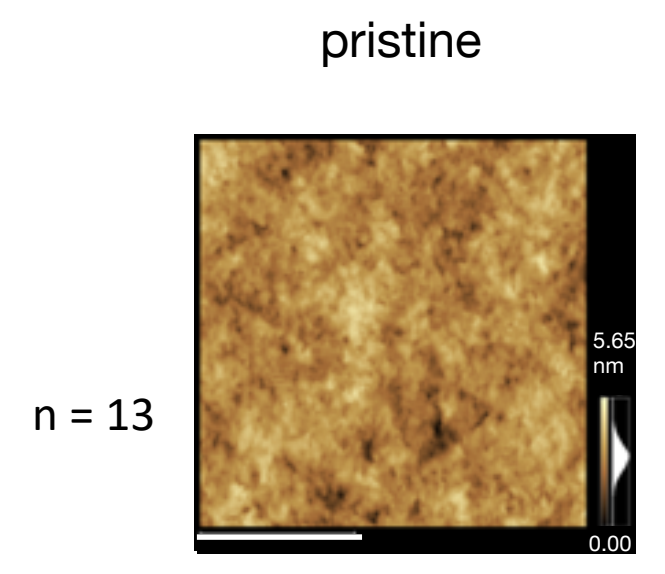

water annealed
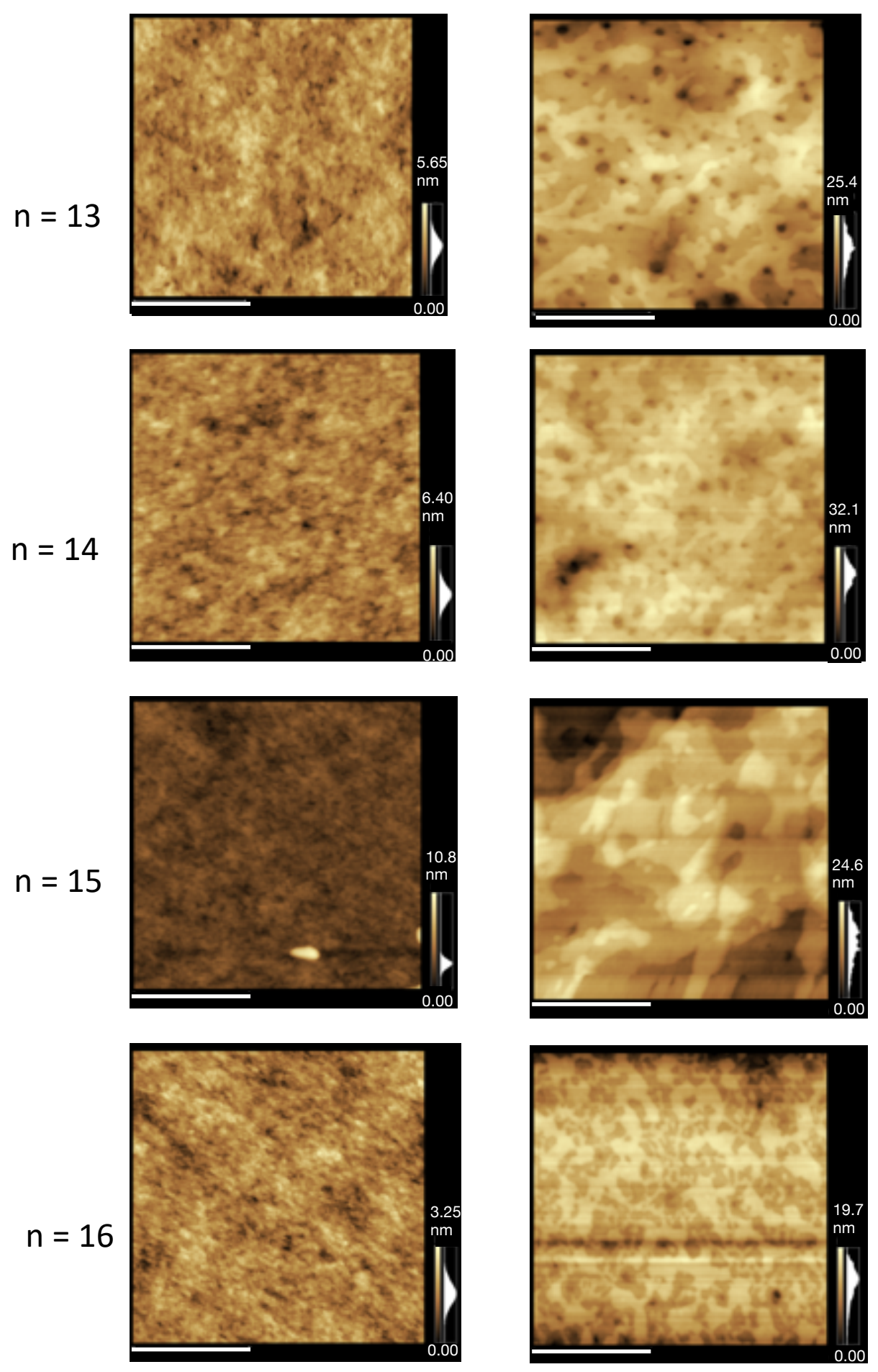

Figure S4 Continued 


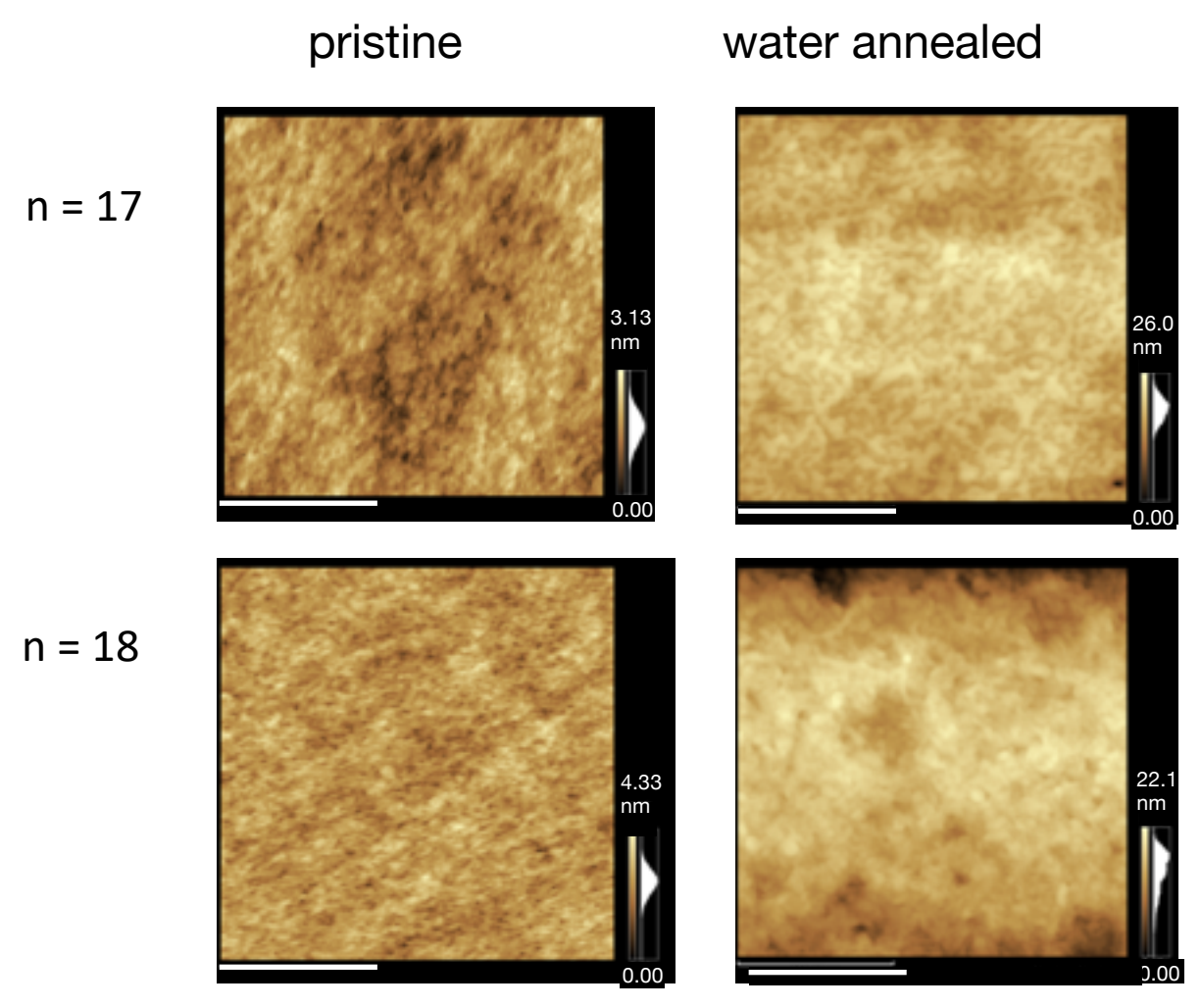

Figure S4 Continued 